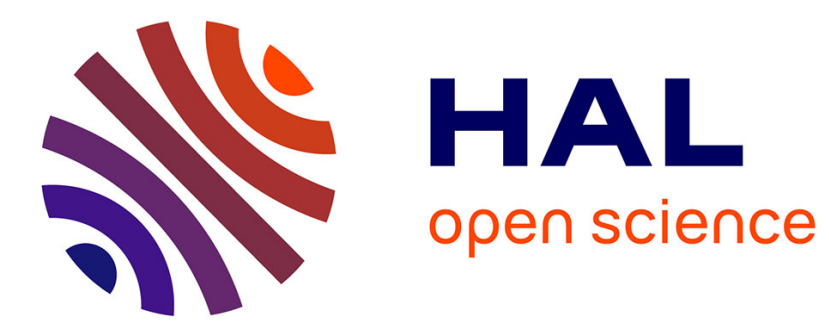

\title{
Explanation of dominant oblique radio emission at Jupiter and comparison to the terrestrial case
}

Fabrice Mottez, Sébastien Hess, Philippe Zarka

\section{To cite this version:}

Fabrice Mottez, Sébastien Hess, Philippe Zarka. Explanation of dominant oblique radio emission at Jupiter and comparison to the terrestrial case. Planetary and Space Science, 2010, 58 (10), pp.14141422. 10.1016/j.pss.2010.05.012 . hal-00658856

\section{HAL Id: hal-00658856 https://hal.science/hal-00658856}

Submitted on 16 Jan 2012

HAL is a multi-disciplinary open access archive for the deposit and dissemination of scientific research documents, whether they are published or not. The documents may come from teaching and research institutions in France or abroad, or from public or private research centers.
L'archive ouverte pluridisciplinaire HAL, est destinée au dépôt et à la diffusion de documents scientifiques de niveau recherche, publiés ou non, émanant des établissements d'enseignement et de recherche français ou étrangers, des laboratoires publics ou privés. 


\title{
Explanation of dominant oblique radio emission at Jupiter and comparison to the Terrestrial case
}

\author{
F. Mottez ${ }^{* a}$, S. Hess ${ }^{b}$, P. Zarkac \\ ${ }^{a}$ LUTH, Observatoire de Paris, CNRS, Université Paris Diderot ; 5 Place Jules Janssen, \\ 92190 Meudon, France \\ ${ }^{b}$ LASP, University of Colorado, 1234 Innovation Drive, Boulder 80303, USA \\ ${ }^{c}$ LESIA, Observatoire de Paris, CNRS, UPMC, Université Paris Diderot; 5 Place Jules \\ Janssen, 92190 Meudon, France
}

\begin{abstract}
The Io-Jupiter S-bursts are series of quasi-periodic impulsive decameter radio emissions from the magnetic flux tube connecting Jupiter to its closest galilean satellite Io. This paper discusses the possibility, suggested by previous works by Hess et al, that the S-bursts are triggered by upgoing electrons accelerated (downward) by trapped Alfvén waves, that have mirrored above the Jupiter ionosphere. According to this theory, the S-bursts would correspond to wave modes that propagate at oblique angles with respect to the magnetic field. Oblique propagation is also inferred for the more slowly varying components of Io-Jupiter radio emissions. Previous works, mainly based on observations of the terrestrial AKR, whose generation process is closely related to those of S-bursts, showed that these waves are emitted on perpendicular wave modes. This discrepancy between the Jovian and Terrestrial cases has led to a controversy about the credibility of the S-bursts model by Hess et al. In the present paper, we show that indeed, the most unstable wave modes for Earth AKR, and Io-Jupiter S-bursts, as they are seen from ground based radio-telescopes, are not the same. Several causes are evaluated : observational bias, the different degree of plasma magnetization above Earth and Jupiter, the role of a cold plasma component and of plasma auroral cavities. Furthermore, we make predictions about what kind
\end{abstract}

\footnotetext{
${ }^{*}$ Corresponding author

Email addresses: fabrice.mottez@obspm.fr (F. Mottez), sebastien.hess@obspm.fr (S. Hess), philippe.zarka@obspm.fr (P. Zarka)
} 
of radiation modes a probe crossing the low altitude Io-Jupiter flux tube will see.

Key words: Jupiter-Io interaction S-bursts Radio emissions Electron acceleration Potential drops Earth AKR

\section{Introduction}

The most structured radio emissions from Jupiter are Short-(S-)bursts. They consist of long series of discrete bursts that are 2-shaped in the timefrequency plane. Previous analyses suggest that S-bursts are generated in the Io-Jupiter flux tube, through the cyclotron-maser instability (CMI) of electrons accelerated from Io to Jupiter (Ellis, 1965).

The CMI is also the cause of other Jovian decameter-wave (DAM) radio emissions (Zarka, 1998), as well as that of the Earth Auroral Kilometric Radiation $(\mathrm{AKR})$ that is observed above the Earth ionosphere ( $\mathrm{Wu}$ and Lee, 1979; Wu, 1985; Treumann, 2006). According to the CMI theory, these radiations are emitted at a frequency close to the local electron gyrofrequency $\omega \sim \omega_{c e}$.

It was shown recently that two acceleration mecanisms could build the distribution functions whose instability triggers the S-bursts. These two mecanisms do not exclude each other, probably both of them occur.

The first one, suggested by Ergun et al. (2006); Su et al. (2006, 2007), is acceleration through a quasi-monochromatic inertial Alfvén wave, trapped in the ionospheric resonator (Su et al., 2006) and amplified by the ionospheric feedback (Lysak and Song, 2003). This resonator is a region along the magnetic field lines around the area where the Alfvén wave velocity reaches a minimum value. Hess et al. (2007a), have evidenced the effect of Alfvénic acceleration in a numerical simulation. The motion of the electrons in the wave electric field and the up and downgoing electron distribution functions at various times and altitudes were computed. They were used to evaluate the CMI growth rate as a function of time and altitude/frequency. This simulation allowed to reproduce the main global features of the S-bursts time-frequency spectrum: discreteness, shape, frequency drift and repetition.

The acceleration process occuring in the Io flux tube has been extensively studied by Hess et al. (submitted). The authors included in their study the computation of the transmission of the Alfvén waves between Io and Jupiter, and the energy gain of the electrons using several theoretical models and 
simulations (including those of Hess et al. (2007a)) and compared it to the latest electromagnetic observations from UV to radio. They conclude that small scale Alfvén waves generated by filamentation of a mother long-scale Alfvén wave are responsible for the acceleration of the electrons in the Io flux tube.

The second mecanism is downward acceleration by electrostatic structures. It has been first evidenced through an analysis of finer structures in the S-bursts time-frequency spectrum (Hess et al., 2007b, 2009a,b). These structures have been observed drifting upward at the local sound speed, thus consistent with ion acoustic solitary waves, or strong double layers.

We call, thoughout this paper: "Hess model" the evidence of Alfvénic and electrostatic acceleration infered from the above mentioned analysis of the S-bursts radio emissions.

These two acceleration mecanisms are well known on Earth, and it seems therefore important to compare, for the two planets, the effect of the accelerated electrons on the CMI and on the radio emissions produced. The works of Hess et al. provide the first observational (indirect) evidence of such acceleration mecanisms outside the terrestrial environment. But with the Hess model, things do not seem to happen exactly as in the case of the terrestrial AKR.

According to this model, as will be recalled in details in section 3 , the waves emitted from the Jupiter-Io flux tube have an oblique angle of propagation with respect to the local magnetic field at the source. This result contrasts with the near-perpendicular beaming observed for the Earth AKR and with the wide-spread idea that oblique waves are much less unstable than those propagating perpendicular to the magnetic field at the source. By analogy with the Terrestrial case, we should expect to observe dominant perpendicularly propagating waves at Jupiter. The fact that the Hess model favours oblique waves makes it a-priori less convincing. We discuss here this specific question and propose several arguments in favour of the dominant amplification of oblique waves in the Jovian radiosources plasma.

The main question addressed in this paper is therefore: "Why do oblique modes correspond to the observable features of the Io-Jupiter decameter radiation while the experience acquired from terrestrial studies indicates that perpendicular modes should be more unstable?"

After defining precisely the radio components that we compare (section 2 ), we recall that several independent studies by Hess et al. conclude to the generation of unstable oblique waves (section 3). Then, we are led to address 
the following points : when comparing the Earth's AKR with the Io-Jupiter decameter radiation (Io-DAM), is there a bias imposed by the conditions of observation that would favour the observations of oblique waves at Jupiter only ? (Section 4.) In order to go further, it is necessary to recall fundamentals of wave dipersion (section 5), resonance condition (section 6), and CMI growth rates (section 7). The Earth AKR is emitted almost exclusively from plasma cavities. Is the role of the plasma cavities likely to be the same in the terrestrial and Jovian environments ? What is their connection with acceleration regions? (Section 8.) Do the same wave modes propagate in the Earth cavities and above Jupiter? We discuss in particular wave propagation outside their source regions (section 9). Finally, we make some predictions about what will be measured by future space instruments, according to the regions explored, or by low-frequency radiotelescopes observing below the Earth's ionospheric cutoff (section 10). These predictions are the ultimate validity tests of the Hess model.

\section{Which AKR components compare to the Io-Jupiter S-bursts ?}

On Earth, the AKR often seems unstructured in the time-frequency plane. Analyzing the AKR fine structure observed with the FAST spacecraft, Pottelette et al. (2001) found a few occurrences of narrow band drifting structures of two kinds. Some of these structures are moving with a velocity in the range 10-100 km/s, typical of ion acoustic structures. Mutel et al. (2006) showed similar evidences of narrow-band structured emissions with strong frequency drifts. They found series of very narrow-band emissions with a negative frequency drift corresponding to upward travelling sources at a speed of a few 100's km/s. These emissions, called "striated AKR" (SAKR) have a frequency of occurence of the order of one percent. Mutel et al. showed that their sources have a spatial extent along the magnetic field that does not exceed $\sim 10 \mathrm{~km}$. Numerical simulations have shown that the SAKR could be triggered, through the CMI, by a bunch of upward propagating ion holes (Mutel et al., 2007). Could the Io-Jupiter (fast drifting) S-bursts be triggered by ion acoustic structures or ion holes ? There are two arguments against it. First, measurements of the S-burst source velocity along the magnetic field lines show that it is consistent with the adiabatic motion of keV electrons, i.e. far higher (100's of times) than the velocity of ion structures. Second, the S-burst bandwidths imply that the sources of emission can have an overall spatial extent of several hundreds of kilometers, 
far more than the expected size of SAKR sources, but consistent with the size of an electron beam travelling at $>0.1 \mathrm{c}$ after acceleration by an Alfvén wave whose frequency is a few tens of $\mathrm{Hz}$.

The second kind of fine structures found by Pottelette et al. (2001) consists of a relatively weak AKR background actually made of the superposition of many fast drifting structures, corresponding to source velocities of $300-1000 \mathrm{~km} / \mathrm{s}$. These velocities are characteristic of electron acoustic structures, and close to the thermal electron velocity. In that sense, these narrowband fast drifting structures bear similarities with the Jovian S-bursts. But S-bursts are discrete structures with a repetition rate about $15 \mathrm{~Hz}$, while the above fine AKR structures form an almost continuous background. According to Pottelette et al. (2001) and Treumann et al. (2008), these AKR structures are associated with electron holes, whose typical size is of the order of a few Debye lengths. According to the "Hess model", S-bursts are rather associated with larger sites of acceleration associated to Alfvén waves. Moreover, the CMI emission associated with electron holes is associated to perpendicular R-X waves, as the other terrestrial AKR sources, while this is likely not to be the case for Io-Jupiter S-burst, as demonstrated in the next sections. Finally, Su et al. (2008) have found in FAST data eight occurences of AKR emission contituted of fast drifting ion acoustic solitons, similar to the SAKR sources but with faster velocities (see McFadden et al., 2003). Emissions occur in that case quasi-periodically with a repetition frequency of 7-18 Hz, similar to the frequency of the Alfvén waves recorded simultaneously by FAST. Moreover the measurements of the electron distributions related to these events are very similar to those simulated by Su et al. (2007) and Hess et al. (2007a), i.e. ring and shell distributions, which are proof of an Alfvénic acceleration. These Alfvénic driven emissions are localized at a lower altitude $\left(\sim 0.5 R_{E}\right)$ than the SAKRs observed by Mutel et al. (2006) (> $1 R_{E}$ ). The proof, given by $\mathrm{Su}$ et al. (2008) that Alfvén-driven radio emissions exist also on Earth, provides an interesting basis of comparison with the S-burst theory proposed by Hess et al. (2007a). Nevertheless, in the present paper we have not based our comparison of Jovian S-bursts and AKR on these Alfvén-driven terrestrial fine structures, due to their rarity. We rather compare below the most common form of AKR on Earth, which shows no obvious fine structures (or a background of fast drifting structures as in Pottelette et al. (2001)) with the most common form of S-bursts from the Io-Jupiter flux tube. 


\section{Generation of oblique modes at Jupiter}

The main difference between the generation of terrestrial AKR and the Hess model of Io-Jupiter S-Bursts is the angle of propagation of the unstable radio waves, whih is related to the distribution functions of the emitting electrons.

At Jupiter, the S-bursts are found to originate from oblique unstable waves triggered by transient electron hollow beams (or rings). Figure 1 (from (Hess et al., 2007a)) shows three electron distribution functions. At the top, we can see an idealized shell distribution function combined with a loss cone, also called a horseshoe distribution. The two other distributions result from a simulation of the electron motion along the Io-Jupiter flux tube in the presence of an Alfvén wave. They are taken at two different times, at the same altitude of $0.1 R_{J}$ above the surface of the planet, within the S-burst source region, well below the acceleration region associated with the Alfvén wave electric field (i.e $>0.9 R_{J}$ in this simulation, or $>0.5 R_{J}$ in the model of $\mathrm{Su}$ et al. $(2006,2007)$. We can see that these distributions are not static. They are not spatially uniform either. This time and space dependence is quasi-periodic, and depends on the Alfvén wave periodicity. On the middle panel, the prominent feature is a partial shell made of downgoing electrons. On the bottom panel, we can see the temporary formation of a hollow beam (i.e. a ring distribution) of upgoing electrons that have mirrored at a lower altitude. These two distributions, ring and shell, are known to be unstable to the CMI. Similar distributions have been observed at Earth by the instruments onboard the FAST spacecraft (Ergun et al., 2006).

Hess et al. (2007a, 2009a) have shown that a transient ring or loss cone distribution can explain the S-burst time-frequency structure. In particular, they have successfully reproduced the typical shape of S-bursts dynamic spectra recorded in Nançay by a simulation of a dynamic spectrum based on the CMI amplification of oblique waves driven by a ring distribution (see Fig. 2 of (Hess et al., 2007a) and Fig. 1 of (Hess et al., 2009a)).

S-bursts are not the only Io-Jupiter radiation that is emitted obliquely. It is also the case of Io-DAM arcs, structured on longer timescales $(\sim 1$ hour), and that are also the envelope of S-bursts in the time-frequency plane. Simulating the time-frequency shapes of radio arcs from the Jovian northern (Hess et al., 2008a) and southern (Hess et al., in press) hemispheres as a function of emission beaming, lead angle between the radio emitting field 
line and the instantaneous Io field line, and electron energy, it has been found that they are in all cases emitted as oblique waves. In another paper, the growth rate of the CMI was computed from an electron distribution resulting from an impulsive heating of the plasma by the passage of Io, followed by time of flight effects. It was shown again that the time-frequency signature of the decameter arcs is those of oblique waves (Hess et al., 2008b).

But, according to the CMI theory, there is much more free energy in the shell distribution functions, which trigger perpendicular waves, than in losscone or ring distributions (Pritchett, 1984; Louarn et al., 1990). For Earth's AKR, the most unstable waves in the source region are indeed perpendicular R-X mode triggered by a shell-driven CMI (Delory et al., 1998; Ergun et al., 2000). In the model of Hess et al. (2007a), such perpendicular waves are possible, but they do not exhibit the characteristic time-frequency signature of the S-bursts (see the Fig. 5 of that paper). Actually, the signature of these perpendicularly propagating waves is a dense superposition of upgoing and downgoing structures, that might appear, in a real context, as an unstructured background of emission. This may be similar to the case of the terrestrial AKR mentioned by Pottelette et al. (2001), where a quasicontinuum emission is actually composed of a large number of superimposed discrete structures.

\section{Conditions of observations}

Two factors constrain the possibility of observing the radiations triggered by the CMI. The first one concerns ground-based observations only. It is linked to the Earth ionospheric cutoff frequency, that is approximately 10 $\mathrm{MHz}$. The second one is the requirement for a highly magnetized plasma in the emission region. In the case of a moderately relativistic plasma, with a Lorentz factor $\Gamma \sim 1$, the condition for cyclotron maser resonance implies $\epsilon=\left(\omega_{p e} / \omega_{c e}\right)^{2}<<1$. Let us now comment about these two conditions, in the case of Earth and of the Io-Jupiter flux tube.

The terrestrial AKR cannot be observed from the ground because the sources are situated above the ionosphere, and the emitted frequencies $(\leq 700$ $\mathrm{kHz})$ are well below the ionospheric cutoff $(\sim 10 \mathrm{MHz})$. The AKR has been discovered and observed from space, both inside and outside its sources (Benson and Calvert, 1979; Bahnsen et al., 1989; Roux et al., 1993), with good time and frequency resolutions. Later, these observations have been completed with an analysis of the particles distribution function (Ergun et al., 
2000). Above Earth, the requirement $\epsilon=\left(\omega_{p e} / \omega_{c e}\right)^{2}<<1$ is generally not met in the auroral zone. But small-scale (typically $\leq 100 \mathrm{~km}$ wide) plasma cavities have been observed several times, and well characterized (Hilgers et al., 1992). They are the only places where it is possible to have $\epsilon<<1$. As a matter of fact, all AKR sources crossed until now were located in such cavities (Roux et al., 1993; Ergun et al., 1998a).

The Io-Jupiter S-bursts are observed above $\sim 10 \mathrm{MHz}$ from Earth with radio telescopes such as the decameter arrays in Nançay (Boischot et al., 1980) and Kharkov (Braude et al., 1978) with high time and frequency resolutions. The Io-Jupiter radiation has also been observed in orbit around Jupiter by Galileo (Menietti et al., 1998)), or during flybys by Voyager (Boischot et al., 1981) and Cassini (Zarka et al., 2004)), but with moderate frequency and time resolutions that did not allow to resolve the characteristic time-frequency signature of the S-bursts. Voyager observations nevertheless allowed to identify S-bursts on dynamic spectra down to $5 \mathrm{MHz}$ (Alexander and Desch, 1984). As explained in the previous section, S-bursts observed at high resolutions were successfully modelled by oblique emission down to $\sim 10 \mathrm{MHz}$ (Hess et al., 2007a, 2009a), while Io-DAM arcs were successfully modelled by oblique emission down to a few $\mathrm{MHz}$ (Hess et al., 2008a, in press). At the lowest frequencies, refraction effects must be taken into account (Ray and Hess, 2008). The presence of S-bursts down to $\sim 1 \mathrm{MHz}$ is likely but not yet proven by observations.

A prominent characteristics of Io-DAM emission (arcs) is the limitation of their low-frequency extent down to about $1 \mathrm{MHz}$, whereas $f_{c e}$ goes down to $\sim 60 \mathrm{kHz}$ along the Io flux tube (IFT). Zarka et al. (2001) have explained this limitation by the fact that along the IFT, the requirement $\epsilon=\left(\omega_{p e} / \omega_{c e}\right)^{2} \leq$ 0.01 is only met below an altitude of $\sim 2.5 R_{J}$, and this altitude corresponds indeed to an emission frequency $\omega \sim \omega_{c e} \sim 1 \mathrm{MHz}$ (Fig. 2a,b). The work of Zarka et al. (2001) was based on the empirical Io torus diffusive equilibrium model of Bagenal (1994). More recently, Su et al. (2003) have made numerical models of the plasma acceleration by strong double layers along the IFT. Their simulations include the computation of the electron density along the IFT, and all of them show that it reaches a deep minimum between $\sim 0.5 R_{J}$ and $\sim 1 R_{J}$ above the Jovian ionosphere (Fig. 2c,d), while the previous model placed it about $0.1-0.2 R_{J}$. In Fig. 2 we have plotted the parameter $\epsilon$ along the IFT, computed from the VIP4 internal Jovian magnetic field model (Connerney et al., 1998) and on the densities derived by Bagenal (1994) $(\mathrm{a}, \mathrm{b})$ or by $\mathrm{Su}$ et al. (2003) (c,d), as a function of the abcissa $s$ 
along the flux tube starting at the surface of Jupiter (lower scale) and of the local gyrofrequency deduced from the VIP4 model (upper scale). The distance from the surface of Jupiter to Io along the flux tube is $\sim 7 R_{J}$. On panels $(\mathrm{a}, \mathrm{c})$, the scale of $s$ is linear, emphasizing low-frequencies, while it is logarithmic on panels (b,d), emphasizing high-frequencies. The grey-shaded band represents the range of $\epsilon$ for which CMI is marginally possible and separates regions where CMI works from those where it is quenched. There are three set of five lines on each panel. Each one corresponds to a given equatorial longitude of the magnetic field lines. Dotted lines correspond to magnetic field lines whose longitude is $230^{\circ}$, continuous lines to a longitude of $190^{\circ}$ and dashed lines to $270^{\circ}$. These three longitudes cover the sector of longitudes in which most of the radio emissions are observed (Boischot et al., 1980; Carr et al., 1983; Queinnec and Zarka, 1998). Each line in a same set corresponds to a different solution found by $\mathrm{Su}$ et al. (2003) corresponding to different boundary conditions. We note that the (Su et al., 2003) model suggests that CMI can operate down to $\sim 200 \mathrm{kHz}$, and is thus probably not accurate at large altitudes. But we are primarily concerned with high frequencies (low altitude regions), where the condition $\epsilon \leq 0.01-0.1$ is met in nearly all cases down to the topside ionosphere.

No in-situ measurement at Jupiter has yet allowed to assess the existence of Earth-like plasma cavities, but the plots of Fig. 2 show that at Jupiter, the plasma density is low enough along the IFT so that the value of $\epsilon$ permits CMI emission from $\sim 1 \mathrm{MHz}$ to $40 \mathrm{MHz}$ (upper limit of the Io-DAM, emitted near the planetary surface) even in the absence of any plasma cavity.

\section{Wave propagation angles in cold and warm plasmas}

The dispersion relation of a cold plasma has been studied in the pioneering work of $\mathrm{Wu}$ and Lee (1979). For the case of a purely perpendicular propagation $\left(k_{\|}=0\right)$, the dispersion relation is displayed on the panel $(\mathrm{C})$ of Fig. 3 (adapted from Louarn and Le Quéau (1996b)). The reduced frequency is defined as $\Delta \omega=\left(\omega-\omega_{c e}\right) / \omega_{c e}$. The magnetization parameter $\epsilon=\left(\omega_{p e} / \omega_{c e}\right)^{2}$ corresponds to the value of the "outside cavity" density $n_{o}$ dominated by a high proportion of cold electrons. The electromagnetic modes of interest, that may have a frequency close to the electron gyrofrequency, are the L-O, $\mathrm{R}-\mathrm{X}$ and $\mathrm{Z}$ modes. The L-O and R-X modes behave asymptotically (for large $k_{\perp}$ ) as light waves and can propagate outside the plasma, provided that it is

not too inhomogeneous (this last condition is not fulfilled at the edges of the 
Earth auroral cavities). The L-O and R-X modes have a low frequency cutoff with $\Delta \omega<0$ for the L-O mode (whose cutoff is actually at $\omega_{p e}$ ), and $\Delta \omega>0$ for the R-X mode (actually $\Delta \omega \sim \epsilon$ ). The Z-mode waves have a resonance, becoming electrostatic and trapped for large $k_{\perp}$, with the frequency tending toward an asymptotic value larger than $\omega_{c e}(\sim \epsilon / 2)$.

On Earth, in the sources of AKR, the waves are emitted perpendicular to the magnetic field, i.e. at $k_{\|}=0$. As we have seen, the AKR sources are located in auroral cavities, devoid of cold electrons. In that case, a relativistic correction has to be applied to the electron cyclotron frequency which becomes (and must be replaced everywhere by):

$$
\omega_{c r}=\frac{\omega_{c e}}{\Gamma_{t h}} \sim \omega_{c e}\left(1-\delta_{t h}\right)
$$

where $\Gamma_{t h}$ is determined from $v_{t h^{2}}$, the second order momentum of the electron distribution function, i.e the thermal velocity in the case of a maxwellian distribution (Pritchett, 1984) and $\delta_{t h}=\left(v_{t h}^{2} / 2 c^{2}\right)$. The dispersion relation is displayed on the panels $(\mathrm{A})$ and $(\mathrm{B})$ of Fig. 3. The R-X mode cutoff frequency becomes $\Delta \omega \sim \epsilon-\delta$. The case of panel (A) corresponds to a hot plasma, typical of Earth's plasma cavities, where $\delta>\epsilon$. The case of panel (B) corresponds to a moderately warm plasma $(0<\delta<\epsilon)$, as is maybe the Jovian plasma. The necessary condition for perpendicular instability $(\delta>\epsilon-\delta$, i.e. $\delta>\epsilon / 2)$ is fullfilled in the case of a hot plasma only.

\section{Resonance condition above Jupiter for large oblique angles}

Les us now come back to the case where $k_{\|} \neq 0$. The relativistic resonance condition is then:

$$
\omega=\frac{\omega_{c e}}{\Gamma_{r}}+k_{\|} v_{r \|}
$$

where $\Gamma_{r}>1$ is the relativistic Lorentz factor associated to the velocity of the resonnant electrons $v_{r}$, not necessarily equal to $\Gamma_{t h}$. In the case of perpendicular propagation $\left(k_{\|}=0\right)$, the resonance condition is simply $\omega=$ $\omega_{c e} / \Gamma_{r}$, and corresponds thus to a frequency below the electron gyrofrequency $\omega_{c e}$. It corresponds in the $\left(v_{\|}, v_{\perp}\right)$ plane to a resonance circle centered on the origin, and of radius $v_{r}$, given by:

$$
\left(\frac{v_{r}^{2}}{2 c^{2}}\right)=\delta_{r}=-\Delta \omega
$$


In that case, the most efficient instability is triggered by a particle distribution with a positive velocity gradient $\partial f / \partial v_{\perp}$ along such a circle. This corresponds to a shell distribution function, as displayed on Fig. 1(a,b). A resonance circle corresponding to a perpendicular wave instability is drawn on Fig. 1(a), this is the ("shell r.c.") circle centered on $v_{\|}=v_{\perp}=0$.

Note that the propagation condition $\omega \geq \omega_{c r}(1+\epsilon)$ implies $\Gamma_{r}<\Gamma_{t h}$ (or equivalently $\delta_{r}<\delta_{t h}$ ). This condition defines the fuzzy notion of "hot" plasma: The plasma is sufficiently hot for the CMI to occur on the perpendicular X-mode if the velocity associated with the second order momentum of the electron distribution function (typically the thermal velocity) is larger than the velocity corresponding to the positive gradient $\partial f / \partial v_{\perp}$ in the distribution. This implies the absence of a cold core in the electron distribution function in the radiosource.

Oblique wave instabilities are also possible. They correspond to resonance circles that are not centered on the origin in the velocity space (Wu and Lee, 1979). Such a circle is displayed on Fig. 1(a) ("loss cone r.c."). The instability corresponds to distribution functions where $\partial f / \partial v_{\perp}$ is large somewhere along that circle. Such an instability can be triggered by a hollow beam (i.e. ring) distribution function, as the one displayed on Fig. 1(c). As shown by the analytical calculation below, in the case of the simulations of Hess et al. (2007a), and more generally for the physical conditions inside the IFT, the resonance condition given by eq. 2 allows for relatively large angles of propagation. Zarka et al. (1996) and Hess et al. (2007b, 2009b) have found that the typical energy of the accelerated electrons generating S-bursts is $\sim 4 \mathrm{keV}$. This correspond to a Lorentz factor $\Gamma_{r}=1.0078=0.992^{-1}$ and a velocity $v_{\text {ring }}=0.125 \mathrm{c}$. As in Fig. 1(c), we choose a beam (ring) that corresponds to a pitch angle $\alpha \sim 45^{\circ}$. Then, $k_{\|} v_{\|}=k_{\|} v_{\text {ring }} \cos \alpha \sim 0.088 k_{\|} c$. For an unstable R-X wave, the frequency $\omega$ must be larger than the cutoff, given for a cold plasma core $(\delta=0)$, by $\omega_{R X}=\omega_{c e}(1+\epsilon)$ (Louarn and Le Quéau, $1996 \mathrm{~b})$. With $\theta$ the wave propagation angle, the resonance condition is

$$
\frac{\omega}{\omega_{c e}} \frac{v_{\text {ring }}}{c} N \cos \alpha \cos \theta>\left(1-\frac{1}{\Gamma_{r}}+\epsilon\right) \text {. }
$$

with $\omega / \omega_{c e} \sim 1$ and the X-mode index $N \sim 1$ as long as $\epsilon<<1$. Then numerically,

$$
\cos \theta \geq 11.3 \times\left(\epsilon+7.7 \times 10^{-3}\right) .
$$

We can see from Fig. 2 that $\epsilon$ can be as low as $10^{-6}$. Then, the propagation angles compatible with the resonance condition are limited by $\theta \leq 85^{\circ}$. For 
$\epsilon=10^{-2}$, the propagation angle can still be large $\left(\theta \leq 78^{\circ}\right)$. For $\epsilon=5 \times 10^{-2}$, the propagation angle is more constrained $\left(\theta \leq 49^{\circ}\right)$. The fit of observed arcs made by Hess et al. (2008a), under the assumption of $N \simeq 1$, predicts $\theta \gtrsim 80^{\circ}$ at a distance larger than a few tenth $R_{J}$ from the planet surface (their Fig. 2 and Table 1), in agreement with the above calculation. This is not qualitatively modified when the possibility that $N<1$ is taken into account (Ray and Hess, 2008).

\section{7. $R-X$ mode versus $Z$ mode instability}

In the Earth auroral zone, the AKR is produced by the shell/horseshoe instability in cavities devoid of cold plasma. Its propagation angle is purely perpendicular in the source. The relativistic dispersion function in a hot plasma allows for direct generation of fast $\mathrm{X}$-mode waves perpendicular to the magnetic field.

As we have seen in section 3, according to the Hess model, in the IoJupiter flux tube, the radio waves are directly generated with an oblique propagation angle, resulting from a cyclotron-maser loss-cone/ring instability. There exist also perpendicular waves, more unstable than oblique ones, but they do not exhibit the observed signature of the S-bursts in the timefrequency plane. We propose below a reason why these perpendicular waves are actually not produced.

Le Queau and Louarn (1989) have made an analysis of the relativistic dispersion relation in the case of a ring distribution, for perpendicular propagation. In their computation, the distribution has a null parallel velocity (this is not the case in Hess et al. (2007a)). They have also added a Maxwellian or a cold plasma of arbitrary density with a null velocity. They have shown that in the relativistic case, the unstable complex frequency lies along the circle of radius $\delta\left(\hat{\omega}=\omega_{c e}+i \delta\right)$.

The maximum growth rate is obtained for $\operatorname{Re}(\Delta \omega)=0$. On panels (A) and (B) of Fig. 3, this instability circle has been drawn. We can see that in the case of a hot plasma (panel $(\mathrm{A}), \delta$ large enough), the $\mathrm{X}$-mode is more unstable than the $\mathrm{Z}$ mode. But in the case of a small value of $\delta$ (panel (B)), the maximum growth rate occurs for the Z-mode waves, albeit with a growth rate smaller than that of the X-mode of case (A). In the case of wave propagation supported by a strictly cold plasma (panel $(\mathrm{C})$ ), both the $\mathrm{X}$-mode and the Z-modes are stable. 
In the terrestrial auroral cavities, $\delta$ is large enough so that the $\mathrm{X}$-mode waves are the most unstable ones. In the case of the Jovian S-bursts, according to the Hess model, the propagation of the waves is controlled by the cold plasma (as in the original AKR study by Wu and Lee (1979)) because no cavity is considered (the necessary condition $\epsilon \leq 0.01$ is fulfilled even without cavities). Oblique X-mode waves can be amplified by the CMI,but there is no perpendicular shell instability. However, the Z-mode can be destabilized by a ring-like distribution (consistent with the analyses of Wu and Lee (1979) and Louarn and Le Quéau (1996b)). The panel (B) of Fig. 3, shows the moderately warm plasma dispersion relation for strictly perpendicular waves. We can see that the Z-mode, with frequencies close to $\omega_{c e}$ (i.e. $|\Delta \omega|<\delta$ ) can be destabilized, while the X-mode cannot.

Finally, we conclude that while ring-like distributions can trigger oblique $\mathrm{R}-\mathrm{X}$ waves, they cannot trigger perpendicular $\mathrm{R}-\mathrm{X}$ waves if the propagation of the waves is controlled by a bulk of cold plasma. In that case, only Z-mode waves can propagate at perpendicular angle from the magnetic field.

\section{Acceleration and plasma cavities}

The next question is therefore : why is the wave propagation at Jupiter dominated by the cold plasma, by contrast with the Earth's case ? One answer could be : because the radiation comes from a low altitude plasma, dominated by the Jovian ionosphere that is cold and dense. A look at Fig. 2 shows that the S-burst range of altitudes observable from the ground (i.e. below $0.5-1 R_{J}$ ) is a region of high ionospheric plasma density. But this explanation is not convincing enough because, on Earth, sources of AKR have been observed at very low ionospheric altitudes $(1500 \mathrm{~km})$, where, in general, the plasma is cold and dense too (Mäkelä et al., 1998, 1999). Also, S-bursts may also originate from somewhat higher altitudes. So, what is the difference between Earth AKR and Jovian S-bursts ?

Another explanation concerns the difference of temperature regimes between the Earth AKR and Io-Jupiter S-bursts and the role of the plasma cavities.

Above Earth, the plasma cavities are the source regions of the AKR (Hilgers, 1992). Some of them are large, but most have a small latitudinal extent that does not exceed 100-200 kilometers.

These cavities are not only the places for AKR generation, they are also key regions for plasma acceleration and turbulence. Actually, cavities cross- 
ing by space probes revealed beams of particles with an energy of a few $\mathrm{keV}$, electrostatic coherent structures such as weak double layers and solitary waves (Ergun et al., 1998b; Franz et al., 1998; Cattell et al., 1999; Bounds et al., 1999). The acceleration processes are connected to strong double layers (Mozer et al., 1980; Temerin et al., 1982; Block et al., 1987) or to Alfvén waves (Chmyrev et al., 1989; Louarn et al., 1994). Alfvén waves propagating along the density gradients on the sides of the cavity can trigger parallel electric field, acceleration, electrostatic instabilities and turbulence (Génot et al., 2001a; Génot et al., 2001b, 2004).

The Earth auroral plasma is generally a mixture of cold ionospheric plasma and hot magnetospheric plasma. The cavities are void of cold plasma, they contain only the hot plasma component (Hilgers et al., 1992). The transverse structure of such non-isotherm cavities can be modelized as a tangential discontinuity (Mottez, 2003, 2004). The precise mecanism of formation of the cavities is still under study, but it seems clear that it is associated to the acceleration mechanism itself (Ganguli et al., 1994; Singh et al., 2005). When a plasma has been accelerated in a finite size region, because of its higher velocity, it escapes and the acceleration region is emptied. Of course, it is refilled by the diffusion of the ambiant plasma surrounding the cavity. The more energetic (fastest and hottest) plasma can refill quikly the cavity, but not the slower cold plasma. Therefore, acceleration in a finite size region favors the the formation of a cavity devoid of cold plasma.

The particular case of the electrostatic potential inverted-V structures responsible for most of the electron acceleration at Earth (Melrose et al., 1982; Wu, 1985; Pritchett, 1984; Imhof et al., 2000) can generate elongated cavities along the magnetic field lines. Outside these cavities $\epsilon>0.1$, forbidding any CMI emissions. Hence the Earth AKR is always emitted in/near acceleration regions in cavities filled only with hot plasma.

In the Io flux tube above Jupiter the electron acceleration comes from Alfvén waves and occurs at altitudes above $0.5-1 R_{J}$. This means that, apart from isolated double layers (associated to more complex S-burst features (Hess et al., 2009a)), the source of the S-burst emissions are situated mainly below - not within - the acceleration regions. So, there is no reason why the cold plasma would be removed. As radio emission is possible at Jupiter in the absence of any cavity, since $\epsilon<0.1$ almost everywhere, it is reasonable to assume that, contrary to the case of the Earth AKR, Jovian S-bursts are emitted in regions dominated by a cold and dense plasma component. 
Let us notice that in/near the electrostatic acceleration regions inferred by Hess et al. (2009a) in the IFT, we can expect, as in the terrestrial case, localized plasma cavities devoid of cold plasma. In these localized regions, the relativistic dispersion relation shows that, by contrast with most of the cold plasma regions of the IFT, perpendicular R-X waves could be generated. Hess et al. (2009a) have shown that some characteristics of the S-bursts dynamic spectra (e.g. the dominantly negative drift rates) are associated to oblique R-X modes and electron local acceleration. But we think that less common localized time-frequency structures, not described in (Hess et al., 2009a), having a null or positive frequency drift, could be associated to the perpendicular R-X waves allowed by the presence of a low density hot plasma.

\section{Propagation outside the sources}

The Earth AKR is mainly emitted in the perpendicular direction, on Xmode waves. These waves cannot escape easily from the very low density plasma cavity where they are generated, as it is bordered by sharp density gradients $\left(\nabla_{\perp} n_{e}\right)$. Many papers have been devoted to the way they convert into other modes, propagate and finally escape at higher altitudes. The AKR is reflected along the cavity (against the strong $\nabla_{\perp} n$ ), until it can escape into space, with a different propagation angle (modified through their propagation in a smooth $\nabla_{\|} n$ density gradient, cf. (Mutel et al., 2008)). It is also partially converted into other wave modes (Z, O) accross the cavity boudaries (strong $\left.\nabla_{\perp} n\right)$ (Louarn and Le Quéau, 1996a,b). Various modes were indeed observed outside the sources (Hanasz et al., 2003).

We think that the case of Jupiter is simpler. The S-burst sources are not enclosed in a cavity, and the plasma is probably homogeneous: $\nabla_{\perp} n_{e} \sim 0$, (as far as one agrees with the conclusions of the previous section). In this homogeneous cold plasma, the perpendicular unstable waves must be emitted on the Z-mode. Such a mode cannot propagate freely into the vaccum or a much less dense plasma. As the gradients along the magnetic field are smooth, these Z-mode waves (trapped into the plasma) cannot be converted efficiently into an another mode that would propagate freely and we cannot observe these radiations from Earth.

Conversely, the oblique unstable waves are emitted on the X-mode and can escape freely into space, since the parallel gradients $\nabla_{\|} n_{e}$ are not too high and the $\mathrm{X}$-mode refraction index in the source is already nearly equal 
to 1 . These emissions, highly structured, and associated to upgoing sources only, are the S-bursts that we can observe from Earth.

\section{Perpectives}

We conclude with a few predictions about what should be observed insitu in the Io-Jupiter flux tube, if the Hess model and the above discussion is correct. These predictions constitute a decisive experimental test for this model.

We can expect that the radio experiment onboard Juno (Jupiter polar orbiter), when it will cross the IFT at low altitude (below the acceleration region, i.e $0.5-1 R_{J}$ ) will not see only the oblique waves that are associated to the S-bursts. It will also see a high level of perpendicular waves, emitted on the Z-mode at frequencies slightly below $\omega_{c e}$ (therefore, almost electrostatic). These correspond to the (large) part of the CMI waves that cannot be seen from Earth. From an experimental point of view, the single electric antenna and one-channel receiver on Juno will not allow us to determine the electric field angle with the magnetic field, and they won't provide either the orientation of the incoming wave vector. But the $\mathrm{Z}$ mode can be recognized by the fact that its polarization is strongly electrostatic : its signature on the magnetic antennas should be weak. On the contrary, the X-mode is expected to be electromagnetic.

The waves emitted above $1 R_{J}$, that are not seen from Earth because of the ionospheric cutoff, might show time-frequency structures much more complex than those of the S-bursts. Why ? Because, at these altitudes, the cold plasma is much less dense (favouring of a relativistic dispersion relation) and we expect electron heating and acceleration by Alfvén waves to be present. We have seen that the acceleration regions tend to remove even more the cold plasma. So, at these altitudes comprised between $\sim 1 R_{J}$ and $3.5 R_{J}$ (the upper limit for the condition $\epsilon<<1$ ) the relativistic dispersion relation is that of a hot plasma only. Then, the perpendicular X-mode is probably the most unstable, as is seen in the sources of the Earth AKR. These waves of frequency below the ionospheric cutoff have been observed from spacecraft in the vicinity of Jupiter (Boischot et al., 1981; Alexander and Desch, 1984; Zarka et al., 2004), but the low resolutions of the onboard instruments did not allow to characterize the S-bursts. Besides high-rate observations by Juno or Laplace/EJSM, low-frequency S-bursts might be 
observed by a radiotelescope installed on the far side of the Moon (Basart et al., 1997; Noda et al., 2005).

A summary of our predictions is presented in figure 4 .

\section{Aknowledgment}

The authors wish to thank the Programme National Soleil Terre (PNST) of the INSU/CNRS, for its support in funding this study. We also than R. Ergun and R. Mutel for very useful comments and discussions.

\section{References}

Alexander, J. K., Desch, M. D., May 1984. Voyager observations of Jovian millisecond radio bursts. Journal of Geophysical Research (Space Physics) 89, 2689-2697.

Bagenal, F., Jun. 1994. Empirical model of the Io plasma torus: Voyager measurements. J. Geophys. Res. 99, 11043-11062.

Bahnsen, A., Jespersen, M., Ungstrup, E., Pedersen, B. M., Eliasson, L., Jun. 1989. Viking observations at the source region of auroral kilometric radiation. Journal of Geophysical Research (Space Physics) 94, 6643-6654.

Basart, J. P., Burns, J. O., Dennison, B. K., Weiler, K. W., Kassim, N. E., Castillo, S. P., McCune, B. M., 1997. Directions for Space-Based LowFrequency Radio Astronomy 2. Telescopes. Radio Science 32, 265-276.

Benson, R. F., Calvert, W., Jun. 1979. Isis 1 observations at the source of auroral kilometric radiation. Geophysical Research Letters 6, 479-482.

Block, L. P., Falthammar, C.-G., Lindqvist, P.-A., Marklund, G., Mozer, F. S., Apr. 1987. Electric field measurements on Viking - First results. Geophysical Research Letters 14, 435-438.

Boischot, A., Lecacheux, A., Kaiser, M. L., Desch, M. D., Alexander, J. K., Warwick, J. W., Sep. 1981. Radio Jupiter after Voyager - an overview of the planetary radio astronomy observations. Journal of Geophysical Research $86,8213-8226$. 
Boischot, A., Rosolen, C., Aubier, M. G., Daigne, G., Genova, F., Leblanc, Y., Lecacheux, A., de La Noe, J., Moller-Pedersen, B., Sep. 1980. A new high-gain, broadband, steerable array to study Jovian decametric emission. Icarus 43, 399-407.

Bounds, S. R., Pfaff, R. F., Knowlton, S. F., Mozer, F. S., Temerin, M. A., Kletzing, C. A., 1999. Solitary potential structures associated with ion and electron beams near $1 \mathrm{R}_{E}$ altitude. J. Geophys. Res. 104, 28709-28718.

Braude, S. I., Megn, A. V., Riabov, B. P., Sharykin, N. K., Zhuk, I. N., Mar. 1978. Decametric survey of discrete sources in the Northern sky. I The UTR-2 radio telescope: Experimental techniques and data processing. Astrophysics and Space Science 54, 3-36.

Carr, T. D., Desch, M. D., Alexander, J. K., 1983. Phenomenology of magnetospheric radio emissions. Physics of the Jovian Magnetosphere, pp. 226284 .

Cattell, C. A., Dombeck, J., Wygant, J. R., Hudson, M. K., Mozer, F. S., Temerin, M. A., Peterson, W. K., Kletzing, C. A., Russell, C. T., Pfaff, R. F., Feb. 1999. Comparisons of Polar satellite observations of solitary wave velocities in the plasma sheet boundary and the high altitude cusp to those in the auroral zone. Geophys. Rev. Letters 26, 425-428.

Chmyrev, V. M., Berthelier, A., Berthelier, J. J., Dzhorzhio, N. V., Bosqued, J. M., Jun. 1989. Non-linear Alfven wave generator of auroral particles and ELF/VLF waves. Planetary and Space Science 37, 749-753.

Connerney, J. E. P., Acuña, M. H., Ness, N. F., Satoh, T., Jun. 1998. New models of Jupiter's magnetic field constrained by the Io flux tube footprint. Journal of Geophysical Research (Space Physics) 103, 11929-11940.

Delory, G. T., Ergun, R. E., Carlson, C. W., Muschietti, L., Chaston, C. C., Peria, W., McFadden, J. P., Strangeway, R., Jun. 1998. FAST observations of electron distributions within AKR source regions. Geophysical Research Letters 25, 2069-2072.

Ellis, G. R. A., 1965. The decametric radio emission of Jupiter. Radio Sci. 69D, 1513-1530. 
Ergun, R. E., Carlson, C. W., McFadden, J. P., Delory, G. T., Strangeway, R. J., Pritchett, P. L., Jul. 2000. Electron-Cyclotron Maser Driven by Charged-Particle Acceleration from Magnetic Field-aligned Electric Fields. The Astrophysical Journal 538, 456-466.

Ergun, R. E., Carlson, C. W., McFadden, J. P., Mozer, F. S., Delory, G. T., Peria, W., Chaston, C. C., Temerin, M., Elphic, R., Strangeway, R., Pfaff, R., Cattell, C. A., Klumpar, D., Shelly, E., Peterson, W., Moebius, E., Kistler, L., Jun. 1998a. FAST satellite wave observations in the AKR source region. Geophys. Res. Letter 25, 2061-2064.

Ergun, R. E., Carlson, C. W., McFadden, J. P., Mozer, F. S., Delory, G. T., Peria, W., Chaston, C. C., Temerin, M., Roth, I., Muschietti, L., Elphic, R., Strangeway, R., Pfaff, R., Cattell, C. A., Klumpar, D., Shelley, E., Peterson, W., Moebius, E., Kistler, L., Jun. 1998b. FAST satellite observations of large-amplitude solitary structures. Geophys. Rev. Letters 25, 2041-2044.

Ergun, R. E., Su, Y.-J., Andersson, L., Bagenal, F., Delemere, P. A., Lysak, R. L., Strangeway, R. J., Jun. 2006. S bursts and the Jupiter ionospheric Alfvén resonator. Journal of Geophysical Research (Space Physics) $111(10), 6212-+$.

Franz, J. R., Kintner, P. M., Pickett, J. S., 1998. POLAR observations of coherent electric field structures. Geophys. Rev. Letters 25, 1277-1280.

Génot, V., Louarn, P., Mottez, F., Dec. 2001b. Fast evolving spatial structure of auroral parallel electric fields. J. Geophys. Res. 106, 29633-29644.

Génot, V., Louarn, P., Mottez, F., Jun. 2004. Alfvén wave interaction with inhomogeneous plasmas: acceleration and energy cascade towards smallscales. Annales Geophysicae 6, 2081-2096.

Ganguli, S. B., Mitchell, H. G., Palmadesso, P. J., Apr. 1994. Auroral plasma transport processes in the presence of $\mathrm{kV}$ potential structures. Journal of Geophysical Research (Space Physics) 99 (18), 5761-5770.

Génot, V., Mottez, F., Louarn, P., Jan. 2001a. Particle Acceleration Linked to Alfven Wave Propagation on Small Scale Density Gradients. Physics and Chemistry of the Earth C 26, 219-222. 
Hanasz, J., Panchenko, M., de Feraudy, H., Schreiber, R., Mogilevsky, M. M., Nov. 2003. Occurrence distributions of the auroral kilometric radiation ordinary and extraordinary wave modes. Journal of Geophysical Research (Space Physics) 108, 1408--.

Hess, S., Cecconi, B., Zarka, P., Jul. 2008a. Modeling of Io-Jupiter decameter arcs, emission beaming and energy source. Geophys. Res. Lett. 35, 13107+ .

Hess, S., Mottez, F., Zarka, P., Nov. 2007a. Jovian S burst generation by Alfvén waves. Journal of Geophysical Research (Space Physics) 112 (11), $11212-+$.

Hess, S., Mottez, F., Zarka, P., Jul. 2009a. Effect of electric potential structures on Jovian S-burst morphology. Geophysical Research Letters 36, $14101-+$.

Hess, S., Mottez, F., Zarka, P., Chust, T., Mar. 2008b. Generation of the jovian radio decametric arcs from the Io Flux Tube. Journal of Geophysical Research (Space Physics) 113, 3260-3281.

Hess, S., Zarka, P., Mottez, F., Jan. 2007b. Io-Jupiter interaction, millisecond bursts and field-aligned potentials. Planetary and Space Science 55, 89-99.

Hess, S., Zarka, P., Mottez, F., Ryabov, V. B., Jan. 2009b. Electric potential jumps in the Io-Jupiter flux tube. Planetary and Space Science 57, 23-33.

Hess, S. L. G., Delamere, P. E., Dols, V., Bonfond, B., Swift, D., submitted. Power transmission and particle acceleration along the Io flux tube. Journal of Geophysical Research.

Hess, S. L. G., Petin, A., Zarka, P., Cecconi, B., Bonfond, B., in press. Modeling the Io-controled Radio-arcs: Lead angle and particle velocity measurements. Planetary and Space Sciences.

Hilgers, A., Feb. 1992. The auroral radiating plasma cavities. Geophys. Res. Lett. 19, 237-240.

Hilgers, A., Holback, B., Holmgren, G., Bostrom, R., Jun. 1992. Probe measurements of low plasma densities with applications to the auroral acceleration region and auroral kilometric radiation sources. Journal of Geophysical Research (Space Physics) 97 (16), 8631-8641. 
Imhof, W. L., Walt, M., Anderson, R. R., Chenette, D. L., Hawley, J. D., Mobilia, J., Petrinec, S. M., Jan. 2000. Association of electron precipitation with auroral kilometric radiation. Journal of Geophysical Research 105, $277-290$.

Le Queau, D., Louarn, P., Mar. 1989. Analytical study of the relativistic dispersion - Application to the generation of the auroral kilometric radiation. Journal of Geophysical Research (Space Physics) 94, 2605-2616.

Louarn, P., Le Quéau, D., Mar. 1996a. Generation of the Auroral Kilometric Radiation in plasma cavities-I. Experimental study. Planetary and Space Science 44, 199-210.

Louarn, P., Le Quéau, D., Mar. 1996b. Generation of the Auroral Kilometric Radiation in plasma cavities-II. The cyclotron maser instability in small size sources. Planetary and Space Science 44, 211-224.

Louarn, P., Roux, A., de Feraudy, H., Le Queau, D., Andre, M., May 1990. Trapped electrons as a free energy source for the auroral kilometric radiation. J. Geophys. Res. 95, 5983-5995.

Louarn, P., Wahlund, J. E., Chust, T., de Feraudy, H., Roux, A., Holback, B., Dovner, P. O., Eriksson, A. I., Holmgren, G., 1994. Observation of kinetic Alfven waves by the Freja spacecraft. Geophys. Res. Lett. 21, 1847-+.

Lysak, R. L., Song, Y., Feb. 2003. Kinetic theory of the Alfvén wave acceleration of auroral electrons. Journal of Geophysical Research (Space Physics) 108 (A4), 6-1.

Mäkelä, J. S., Mälkki, A., Koskinen, H., Boehm, M., Holback, B., Eliasson, L., May 1998. Observations of mesoscale auroral plasma cavity crossings with the Freja satellite. Journal of Geophysical Research (Space Physics) 103 (12), 9391-9404.

Mäkelä, J. S., Mälkki, A. M., Koskinen, H. E. J., Clemmons, J. H., Erlandson, R. E., Holback, B., Eliasson, L., Aug. 1999. Evolution of mesoscale auroral cavities before substorm onset. Journal of Geophysical Research (Space Physics) 104 (13), 17201-17216. 
McFadden, J. P., Carlson, C. W., Ergun, R. E., Mozer, F. S., Muschietti, L., Roth, I., Moebius, E., Apr. 2003. FAST observations of ion solitary waves. Journal of Geophysical Research (Space Physics) 108, 8018-+.

Melrose, D. B., Hewitt, R. G., Ronnmark, K. G., Jul. 1982. Terrestrial kilometric radiation - The cyclotron theory. Journal of Geophysical Research $87,5140-5150$.

Menietti, J. D., Gurnett, D. A., Kurth, W. S., Groene, J. B., Granroth, L. J., Sep. 1998. Galileo direction finding of Jovian radio emissions. Journal of Geophysical Research 103, 20001-20010.

Mottez, F., Jun. 2003. Exact nonlinear analytic Vlasov-Maxwell tangential equilibria with arbitrary density and temperature profiles. Physics of Plasmas 10, 2501-+.

Mottez, F., Sep. 2004. The pressure tensor in tangential equilibria. Annales Geophysicae 22 (9), 3033-3037.

Mozer, F. S., Cattell, C. A., Hudson, M. K., Lysak, R. L., Temerin, M., Torbert, R. B., 1980. Satellite measurements and theories of low altitude auroral particle acceleration. Space Science Reviews 27, 155-213.

Mutel, R. L., Christopher, I. W., Pickett, J. S., Apr. 2008. Cluster multispacecraft determination of AKR angular beaming. Geophysical Research Letters 35, 7104-+.

Mutel, R. L., Menietti, J. D., Christopher, I. W., Gurnett, D. A., Cook, J. M., Oct. 2006. Striated auroral kilometric radiation emission: A remote tracer of ion solitary structures. Journal of Geophysical Research (Space Physics) 111 (A10), 10203-+.

Mutel, R. L., Peterson, W. M., Jaeger, T. R., Scudder, J. D., Jul. 2007. Dependence of cyclotron maser instability growth rates on electron velocity distributions and perturbation by solitary waves. Journal of Geophysical Research (Space Physics) 112 (A11), 7211-+.

Noda, H., Hanada, H., Kawano, N., Iwata, T., Jun. 2005. The SELENE Mission and Japanese Lunar Exploration Scenario. Journal of Korean Astronomical Society 38, 311-314. 
Pottelette, R., Treumann, R. A., Berthomier, M., May 2001. Auroral plasma turbulence and the cause of auroral kilometric radiation fine structure. Journal of Geophysical Research (Space Physics) 106, 8465-8476.

Pritchett, P. L., Feb. 1984. Relativistic dispersion and the generation of auroral kilometric radiation. Geophys. Res. Letters 11, 143-146.

Queinnec, J., Zarka, P., Nov. 1998. Io-controlled decameter arcs and IoJupiter interaction. Journal of Geophysical Research (Space Physics) 103, 26649-26666.

Ray, L. C., Hess, S., Nov. 2008. Modelling the Io-related DAM emission by modifying the beaming angle. Journal of Geophysical Research (Space Physics) 113 (A12), 11218-+.

Roux, A., Hilgers, A., de Feraudy, H., Le Queau, D., Louarn, P., Perraut, S., Bahnsen, A., Jespersen, M., Ungstrup, E., Andre, M., Jul. 1993. Auroral kilometric radiation sources - In situ and remote observations from Viking. Journal of Geophysical Research (Space Physics) 98, 11657-+.

Singh, N., Deverapalli, C., Khazanov, I., Jul. 2005. Comparison of electric fields and density structures seen in simulations and satellite observations. Geophys. Res. Lett. 32, 13108.

Su, Y., Ergun, R. E., Jones, S. T., Strangeway, R. J., Chaston, C. C., Parker, S. E., Horwitz, J. L., Jun. 2007. Generation of short-burst radiation through Alfvénic acceleration of auroral electrons. Journal of Geophysical Research (Space Physics) 112 (A11), 6209-+.

Su, Y., Ma, L., Ergun, R. E., Pritchett, P. L., Carlson, C. W., Aug. 2008. Short-burst auroral radiations in Alfvénic acceleration regions: FAST observations. Journal of Geophysical Research (Space Physics) 113 (A12), $8214-+$.

Su, Y.-J., Ergun, R. E., Bagenal, F., Delamere, P. A., Feb. 2003. Io-related Jovian auroral arcs: Modeling parallel electric fields. Journal of Geophysical Research (Space Physics) 108, 1094-+.

Su, Y.-J., Jones, S. T., Ergun, R. E., Bagenal, F., Parker, S. E., Delamere, P. A., Lysak, R. L., Jun. 2006. Io-Jupiter interaction: Alfvén wave propagation and ionospheric Alfvén resonator. J. Geophys. Res. 111 (A10), $6211-+$. 
Temerin, M., Cerny, K., Lotko, W., Mozer, F. S., Apr. 1982. Observations of double layers and solitary waves in the auroral plasma. Physical Review Letters 48, 1175-1179.

Treumann, R. A., Aug. 2006. The electron cyclotron maser for astrophysical application. Astron. Astrophys. Review 13, 229-315.

Treumann, R. A., Jaroschek, C. H., Pottelette, R., Dec. 2008. On deformation of electron holes in phase space. Europhysics Letters 84, 69001-+.

Wu, C. S., Aug. 1985. Kinetic cyclotron and synchrotron maser instabilities Radio emission processes by direct amplification of radiation. Space Science Reviews 41, 215-298.

Wu, C. S., Lee, L. C., Jun. 1979. A theory of the terrestrial kilometric radiation. Astrophysical Journal 230, 621-626.

Zarka, P., Sep. 1998. Auroral radio emissions at the outer planets: Observations and theories. Journal of Geophysical Research (Space Physics) 103, 20159-20194.

Zarka, P., Cecconi, B., Kurth, W. S., Aug. 2004. Jupiter's low-frequency radio spectrum from Cassini/Radio and Plasma Wave Science (RPWS) absolute flux density measurements. J. Geophys. Res. 109, A09S15.

Zarka, P., Farges, T., Ryabov, B. P., Abada-Simon, M., Denis, L., 1996. A scenario for Jovian S-bursts. Geophys. Res. Letters 23, 125-128.

Zarka, P., Queinnec, J., Crary, F. J., Aug. 2001. Low-frequency limit of Jovian radio emissions and implications on source locations and Io plasma wake. Planetary and Space Science 49, 1137-1149. 

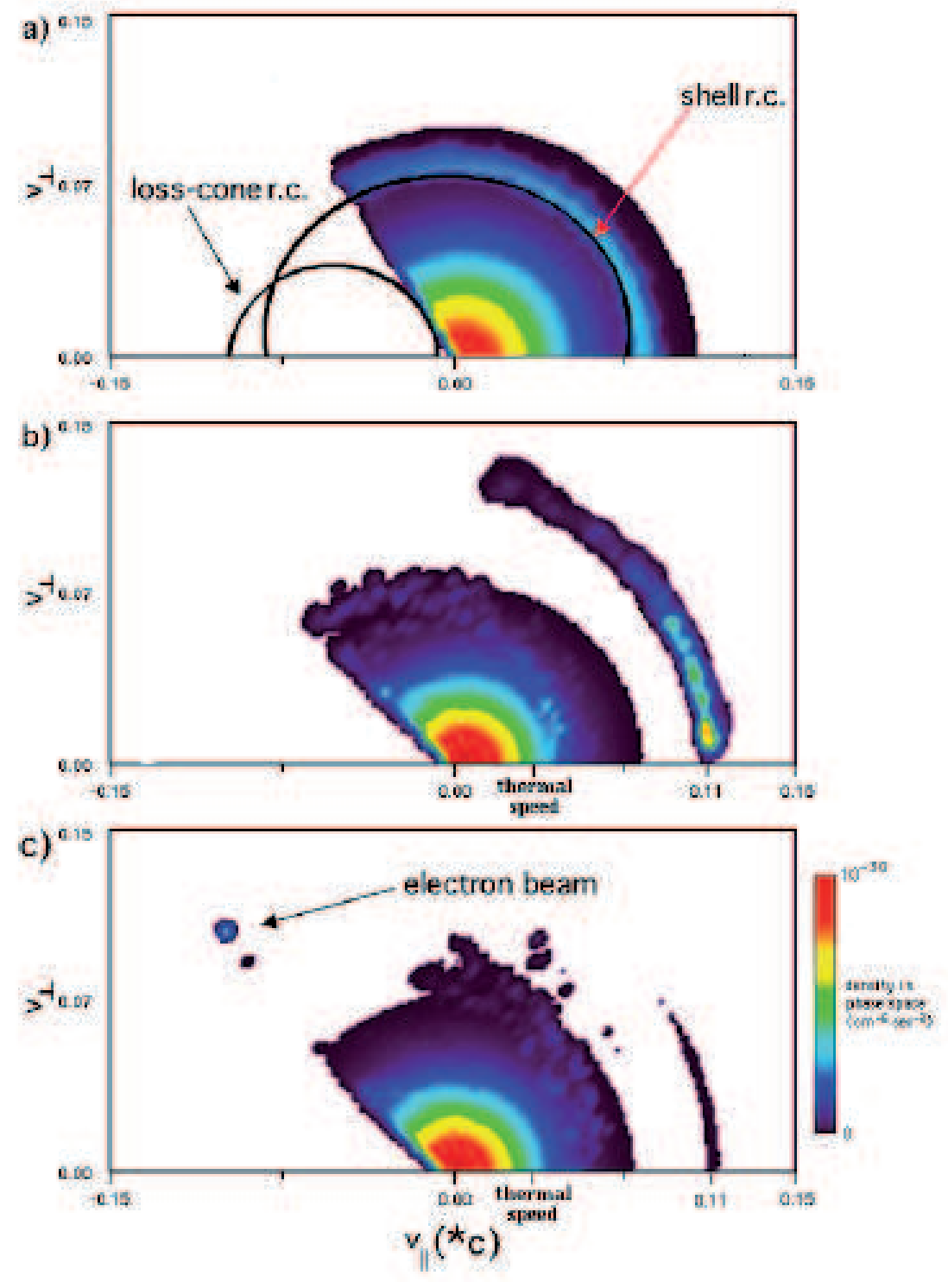

Figure 1: (a) Example of an unstable electron distribution relatively to the cyclotronmaser instability. The "loss cone" is due to magnetic mirror effect and loss by collisions in the ionosphere. The perpendicular gradient is positive along the loss cone border, and the resonance circle (r.c.) is tangent to it. A "shell" distribution is a ring of particles in the phase space. The resonance circle is tangent to its inner edge. (b and c) Distributions of the particles at different time of the simulation at an altitude of $0.1 R_{J}$. Planetward accelerated electrons form an arc in the $\left(v_{\|}, v_{\perp}\right)$ plane because of adiabatic motion and magnetic mirroring (panel b). This arc is cut by the loss cone, generating antiplanetward electron beams moving adiabatically. Thes2 5 eams are nearly aligned with the loss cone border (panel c). From Hess et al. (2007a) 

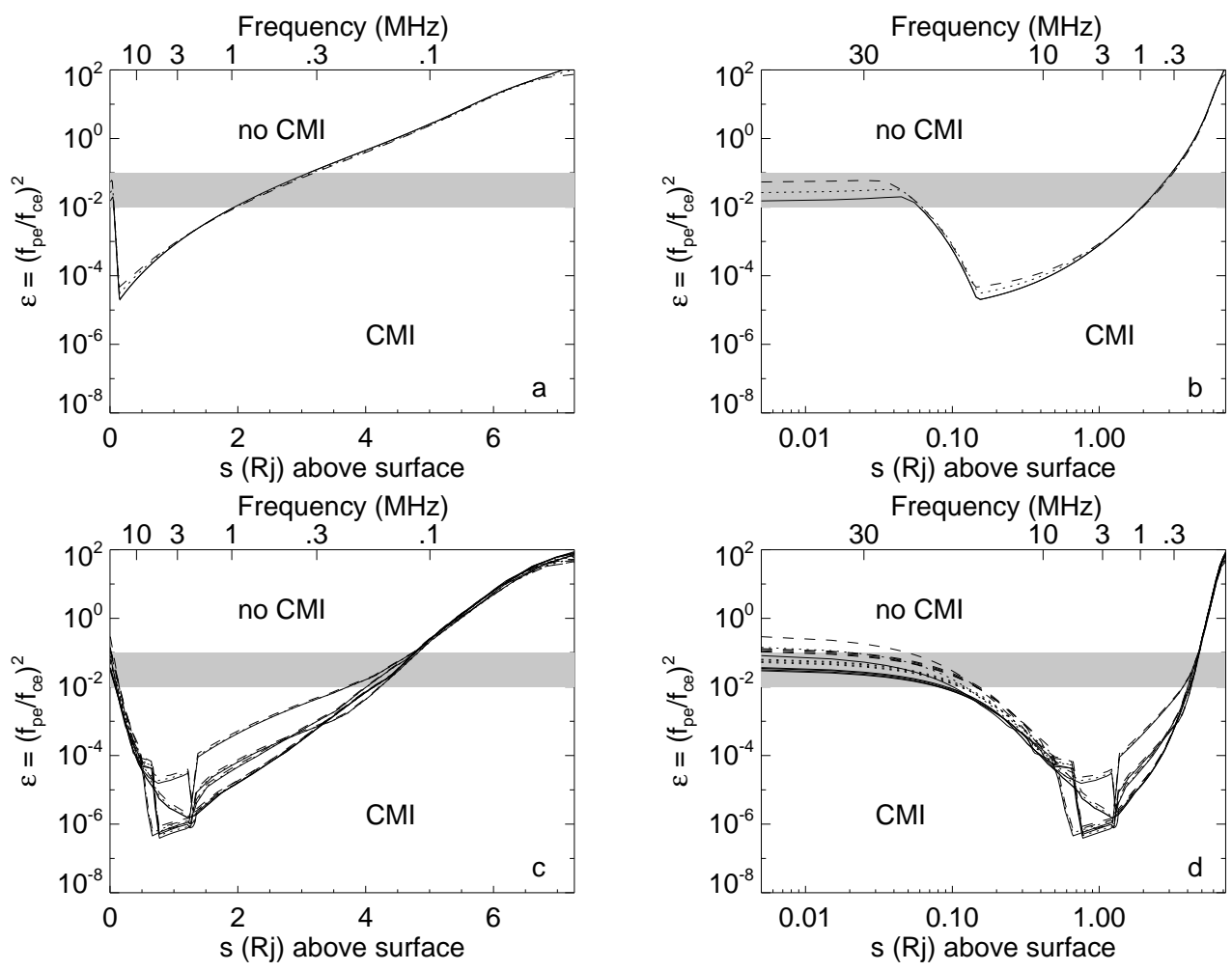

Figure 2: The magnetization parameter $\epsilon$ as a function of curvilinear abcissa $s$ along the Io-Jupiter flux tube and as a function of the corresponding gyrofrequency. Panels $(a, b)$ were computed as in (Zarka et al., 2001), based on the torus model of Bagenal (1994). Panels (c,d) were computed using the numerical models of Su et al. (2003). On panels (a,c), the linear scale of $s$ emphasizes low-frequencies, while it is logarithmic on panels (b,d), emphasizing high-frequencies. The grey-shaded band represents the range of $\epsilon$ for which CMI is marginally possible and separates regions where CMI works from those where it is quenched. More details are given in section 4. 


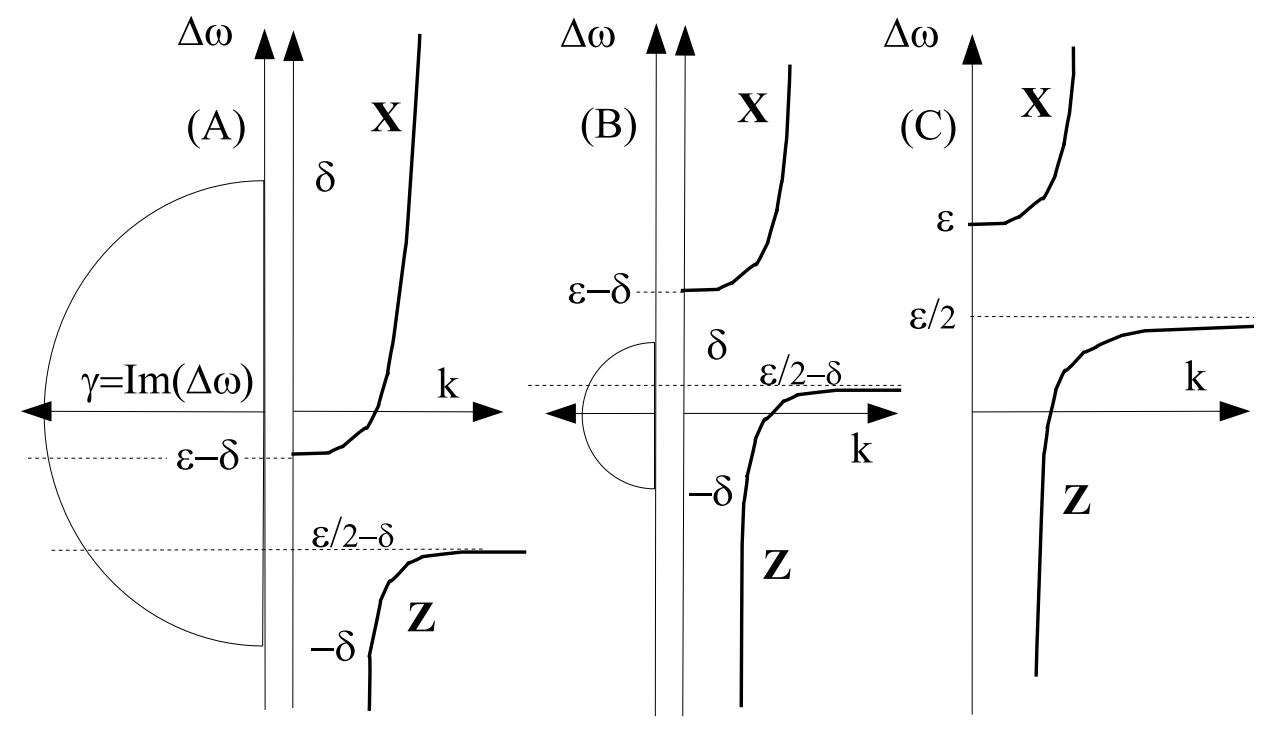

Figure 3: Dispersion relation of the $\mathrm{X}$ and $\mathrm{Z}$ modes for perpendicular propagation. (A) For a hot plasma ring and $\delta>\epsilon ;(\mathrm{B})$ for a hot plasma ring and $\delta<\epsilon ;(\mathrm{C})$ for a cold plasma $(\delta=0)$. Note that $\delta$ here stands for the quantity $\delta_{t h}$ in sections 5 and 6 . The right-hand side axis is the perpendicular wave vector axis. The left-hand side axis is the imaginary part of the reduced frequency, i.e. the growth rate $\Gamma$ of the instability. The vertical axis is the real part of the reduced frequency. A mode is unstable when its real frequency is comprised between the two limits $\delta$ and $-\delta$ of the circle. In case $(\mathrm{A})$ both the $\mathrm{X}$ and $\mathrm{Z}$ modes are unstable, the $\mathrm{X}$ mode being more unstable than the $\mathrm{Z}$ mode. In case (B) only the $\mathrm{Z}$ mode is unstable. In case $(\mathrm{C})$, no mode is unstable : a hot plasma component is required for perpendicular instability. Adapted from Louarn and Le Quéau (1996b). 


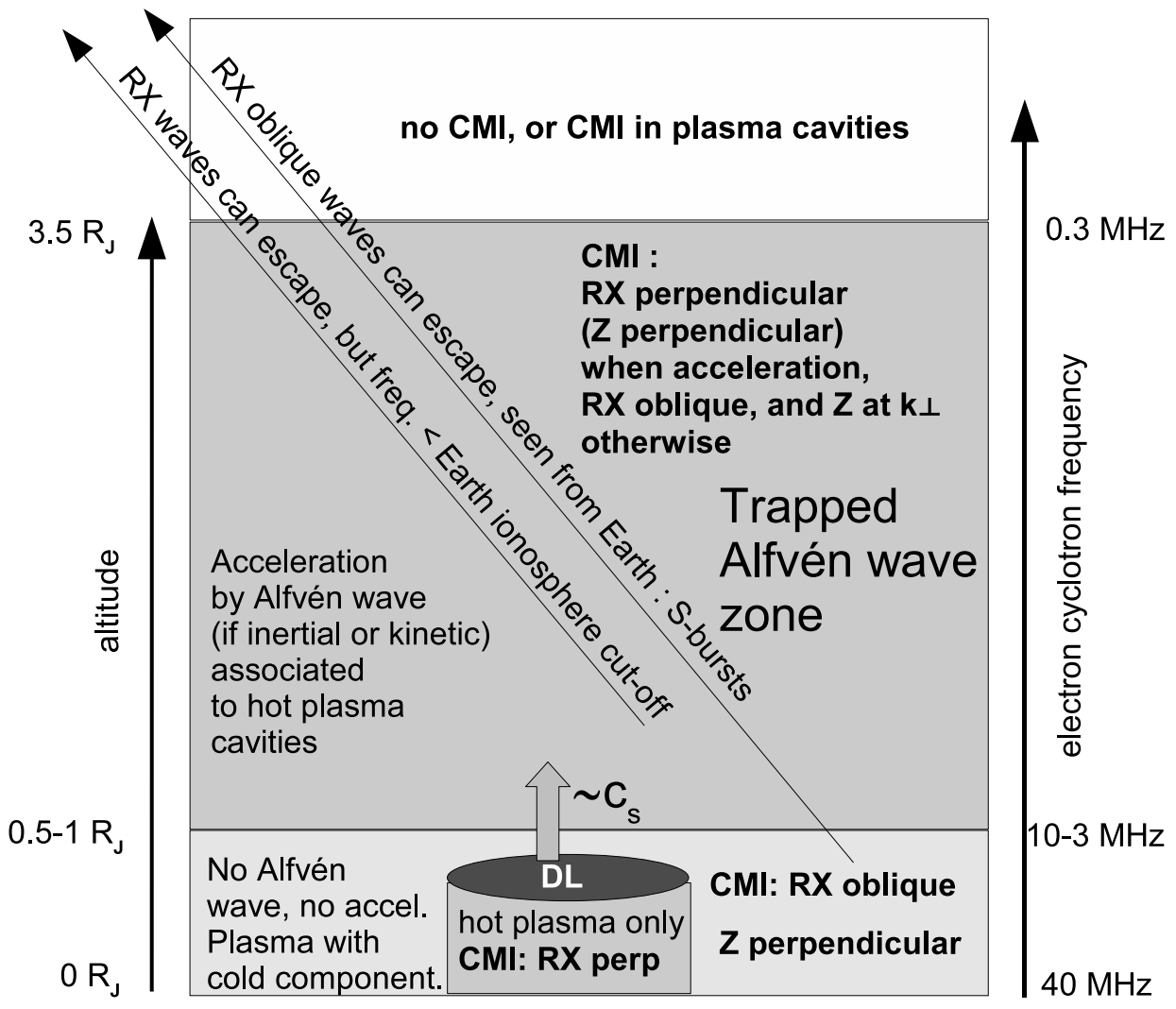

Figure 4: Summary of the acceleration regions, and of the wave modes that might be measured in situ, according to the analysis developed in the present paper. The vertical axis represents the altitude along the IFT, with the corresponding electron cyclotron frequency on the right. Above $3.5 R_{J}, \epsilon$ is large enough for quenching CMI operation. In the region between $0.5-1 R j$ and $3.5 R_{J}, \epsilon<<1$ and Alfvén waves accelerate electrons. Within acceleration region, the plasma is hot, and the CMI favours perpendicular Zmode, and perpendicular and oblique R-X mode waves. Only the oblique R-X waves can propagate directly outside this region, as well as refracted perpendicular R-X mode waves, but they are not observed from Earth because the frequency (less than $10 \mathrm{MHz}$ ) is below the Earth ionospheric cutoff. The region below 0.5-1 $R_{J}$ is below the Alfvén wave resonator, and generally, there is no acceleration. In that case, the plasma is not hot enough to permit perpendicular R-X waves amplification, so that only oblique R-X mode and perpendicular Z-mode are unstable. The R-X oblique mode can propagate in outer space, and is the cause of the highly structured S-bursts observed from Earth. Nevertheless, in this range of altitudes, there are also electrostatic acceleration structures (maybe strong double layers), in the vicinity of which the plasma is hot. From these small regions, perpendicular R-X mode radiations might be seen from Earth. 